\title{
The effects of prior high intensity double poling on subsequent diagonal stride skiing characteristics
}

\author{
Glenn Björklund ${ }^{1 *}$, Hans-Christer Holmberg ${ }^{1,2}$ and Thomas Stöggl ${ }^{1,3}$
}

\begin{abstract}
Purpose: To investigate the influence of prior high intensity double poling (DP) on physiological and biomechanical responses during subsequent diagonal stride (DIA).

Methods: Eight well-trained male cross-country skiers (age $22 \pm 3 \mathrm{yr} ; \mathrm{VO}_{2 \max } 69 \pm 3 \mathrm{ml} \cdot \mathrm{kg}^{-1} \cdot \mathrm{min}^{-1}$ ) roller-skied on a treadmill sequentially for 3 min at 90\% DIA VO ${ }_{2 \max }\left(\mathrm{DIA}_{1}\right), 3$ min at 90\% DP VO $\mathrm{O}_{2 \text { peak }}$ and 3 min at 90\% DIA VO $\mathrm{V}_{2 \max }$ $\left(\mathrm{DIA}_{2}\right)$. Cardio-respiratory responses were monitored continuously and gases and metabolites in blood from the $a$. femoralis, $v$. femoralis and $v$. subclavia determined. Pole and plantar forces and EMG from 6 lower- and upper-body muscles were measured.

Results: $\mathrm{VO}_{2}$ decreased from DIA 1 to DP and increased again to DIA (both $P<0.05$ ), with no difference between the DIA sessions. Blood lactate rose from DIA $A_{1}$ to DP to DIA ${ }_{2}$. $\mathrm{O}_{2}$ extraction was attenuated during $D P(P<0.05)$, but was the same during DIA $A_{1}$ and $D I A_{2}$. EMG ${ }_{R M S}$ for arm muscles during poling phase, as well as peak pole force and cycle rate were higher, while leg muscle activity was lower during DP than both sessions of DIA (all $P<0.05$ ). The ratio of upper-/whole-body EMGRMs correlated negatively with $\mathrm{O}_{2}$ extraction in the arms during both sessions of DIA $(P<0.05)$.

Conclusions: In well-trained skiers skiing at high-intensity DP prior to DIA did not influence $\mathrm{VO}_{2}$, muscle activation or forces in the latter. At race intensity DP does not influence the distribution of work between upper- and lower-body during a subsequent bout of DIA. $\mathrm{O}_{2}$ extraction is coupled to technical skills during skiing.
\end{abstract}

Keywords: EMG; Force; Oxygen extraction; Oxygen uptake

\section{Background}

Of the several different sub-techniques involved in classical cross-country skiing, diagonal stride (DIA) and double poling (DP) have been most frequently studied. With DIA, the skier utilizes both the arms and legs in a diagonal fashion for propulsion and this is considered to be whole-body exercise (Lindinger et al. 2009). In the case of DP, most propulsive force is supplied by the upper-body, although a dynamic leg movement also contributes to the application of body weight to pole forces (Holmberg et al. 2006; Rud et al. 2014). Consequently, in connection with both of these sub-techniques, and particularly DP, recovery of the upper-body muscles between strokes might be limited.

With regards to cardiovascular regulation, with a similar absolute pulmonary oxygen uptake $\left(\mathrm{VO}_{2}\right)$, delivery of

\footnotetext{
* Correspondence: glenn.bjorklund@miun.se

${ }^{1}$ Department of Health Sciences, Swedish Winter Sports Research Centre,

Mid-Sweden University, 83125 Östersund, Sweden

Full list of author information is available at the end of the article
}

$\mathrm{O}_{2}$ to the arms and $\mathrm{VO}_{2}$ are higher, but extraction of $\mathrm{O}_{2}$ by the arms lower with DP than DIA (Calbet et al. 2005). In the case of DIA, when exercise intensity is reduced from high to moderate, $\mathrm{O}_{2}$ extraction in the arms is lowered to a greater extent than in the legs, probably due to a more pronounced decrease in the activation of arm muscles (Björklund et al. 2010). With DP the opposite is observed, i.e., $\mathrm{O}_{2}$ extraction in the arms is reduced to a lesser extent than in the legs as the intensity of exercise is diminished, apparently because strong activation of the muscles of the upper-body is maintained (Stöggl et al. 2013).

Furthermore, the skeletal muscles of the arms and legs differ with respect to the production and oxidation of lactate, with the arms producing more at a given workload (Ahlborg and Jensen-Urstad 1991). Thus, more extensive upper-body activation during DP compared to DIA should lead to a higher systemic concentration of lactate with the former sub-technique, as has, indeed, 
been shown by Van Hall et al. (2003) to be the case with exercise of moderate intensity $\left(\sim 76 \% \mathrm{VO}_{2 \max }\right)$. In that investigation and the study by Calbet et al. (2005), the cardiovascular and metabolic influence of DP on subsequent DIA were analyzed to a certain extent, but no direct comparisons between DIA before and after DP were made. Moreover, analysis of higher exercise intensities (race intensity) is also important, since the distinctly higher muscle activation and application of force involved might cause mechanical hindrance of $\mathrm{O}_{2}$ extraction and thereby elevate blood levels of lactate (Stöggl et al. 2013).

Whereas DIA and DP have both been characterized individually from a physiological and biomechanical perspective during simulated races (Larsson and HenrikssonLarsen 2005; Mognoni et al. 2001; Mygind et al. 1994; Ortenblad et al. 2011; Welde et al. 2003), the influence of these sub-techniques on one another remains to be examined. Previous investigations on different modes of exercises involving the legs have revealed that the biomechanical and physiological characteristics of running are influenced negatively by prior cycling in the case of moderately trained, but not elite triathletes (Bonacci et al. 2010, 2011). In addition, after this transition, only the latter preserve their running economy, with no change in $\mathrm{VO}_{2}$. With repeated sprints of cross-country skiing the fatigue experienced by the upper-body muscles already during the first sprint leads to a shift in body position and, consequently, less effective application of force during DP (Zory et al. 2009, 2011).

Accordingly, the upper-body muscle fatigue induced by high-intensity DP might alter the relative activation of arm and leg muscles, together with pole and plantar forces, during subsequent high-intensity DIA. Thus, our aim here was to examine the influence of DP on subsequent DIA at a simulated race intensity of $\sim 90 \%$ of $\mathrm{VO}_{2 \text { max/peak }}$ from a biomechanical and physiological perspective. The hypothesis was that DP alters the distribution of work between the upper- and lower-body, i.e., decreases pole force and activation of arm muscles during subsequent DIA, thereby reducing the $\mathrm{O}_{2}$ extraction by the arms.

\section{Methods}

\section{Subjects}

The 8 well-trained male cross-country skiers who volunteered (Table 1) were informed about the test procedures and possible risks prior to providing their written consent to participate. The research techniques and experimental protocol were pre-approved by the Regional Ethical Review Board in Umeå, Sweden (\#08-049M) and performed in accordance with the Declaration of Helsinki.

\section{The general experimental protocol}

All tests were performed on a motor-driven treadmill (Rodby RL 3000, Vänge, Sweden) and, to minimize
Table 1 Anthropometric and physiological characteristics of the 8 well-trained male cross-country skiers who participated

\begin{tabular}{|c|c|}
\hline Variable & Mean \pm SD \\
\hline Age (yr) & $22 \pm 3$ \\
\hline Height (cm) & $183 \pm 4$ \\
\hline Weight (kg) & $79 \pm 6$ \\
\hline $\mathrm{BMI}$ & $23.6 \pm 2.1$ \\
\hline $\mathrm{VO}_{2 \max }\left(\mid \cdot \min ^{-1}\right)$ & $5.4 \pm 0.3$ \\
\hline $\mathrm{VO}_{2 \max }\left(\mathrm{ml} \cdot \mathrm{kg}^{-1} \cdot \mathrm{min}^{-1}\right)$ & $69 \pm 3$ \\
\hline $\mathrm{VO}_{2 \text { peak }}\left(\mid \cdot \mathrm{min}^{-1}\right)$ & $4.8 \pm 0.4$ \\
\hline $\mathrm{VO}_{\text {2peak }}\left(\mathrm{ml} \cdot \mathrm{kg}^{-1} \cdot \mathrm{min}^{-1}\right)$ & $62 \pm 3$ \\
\hline OBLA $\left(\% \mathrm{VO}_{2 \max }\right)$ & $82 \pm 4$ \\
\hline OBLA $\left(1 \cdot \min ^{-1}\right)$ & $4.4 \pm 0.4$ \\
\hline $\mathrm{LT}\left(\% \mathrm{VO}_{2 \max }\right)$ & $76 \pm 3$ \\
\hline $\mathrm{LT}\left(\mid \cdot \mathrm{min}^{-1}\right)$ & $4.1 \pm 0.4$ \\
\hline $\mathrm{HR}_{\max }$ (beats $\cdot \mathrm{min}^{-1}$ ) & $189 \pm 5$ \\
\hline
\end{tabular}

$\mathrm{VO}_{2 \text { max }}$, Maximal $\mathrm{O}_{2}$ uptake; OBLA, onset of blood lactate accumulation (4 mmol. ${ }^{-1}$ ); LT, Lactate threshold; $\mathrm{HR}_{\max }$ Maximal heart rate.

variations in rolling resistance, using the same pair of roller skis (Pro-Ski C2, Sterners, Nyhammar, Sweden). All of the participants were well accustomed to treadmill roller skiing, both as part of their regular training and from their involvement in previous testing. They visited the laboratory on two occasions: a) first, to carry out a preliminary test to determine $\mathrm{VO}_{2 \max }$, maximal heart rate $\left(\mathrm{HR}_{\max }\right)$ and the linearity of the velocity- $\mathrm{VO}_{2}$ relationship for DIA and DP; and b) two days later to perform a continuous experimental protocol involving DIA and DP at race intensity.

\section{The preliminary test}

The velocity required to obtain $90 \% \mathrm{VO}_{2 \max }$ with DIA and $90 \%$ peak oxygen uptake $\left(\mathrm{VO}_{2 \text { peak }}\right)$ with DP were established employing separate linear graded protocols. In the case of DIA, $\mathrm{VO}_{2}$ was determined at several submaximal workloads separated by a one-minute break at a fixed incline of $6.5^{\circ}$, with an initial velocity set to 6 $\mathrm{km} \cdot \mathrm{h}^{-1}$. The increase for the submaximal workloads was $2 \mathrm{~km} \cdot \mathrm{h}^{-1}$ using four minute long bouts. During this submaximal protocol capillary blood samples were taken between workloads for determination of lactate and the onset of blood lactate accumulation (OBLA) $\left(4.0 \mathrm{mmol} \cdot \mathrm{l}^{-1}\right)$ (Ivy et al. 1980; Sjödin and Jacobs 1981). $\mathrm{VO}_{2 \max }$ values were established using a ramp protocol starting at 11 $\mathrm{km} \cdot \mathrm{h}^{-1}$ at an incline of $4^{\circ}$, with an increase in gradient by $1^{\circ}$ every minute. One day later the $\mathrm{VO}_{2 \text { peak }}$ for DP was obtained starting at a fixed inclination of $1^{\circ}$, initial velocity of $17 \mathrm{~km} \cdot \mathrm{h}^{-1}$, and a $2 \mathrm{~km} \cdot \mathrm{h}^{-1}$ increase in workload every fourth minute, until exhaustion. Using the means of the three highest consecutive $\mathrm{VO}_{2}$ values during the last 
minute at each submaximal workload, with a total sampling time of $30 \mathrm{~s}$ (mixing chamber), simple linear regression was applied to calculate the velocities required to achieve the targets of $90 \% \mathrm{VO}_{2 \max }\left(\mathrm{DIA}_{\mathrm{LIN}}\right)$ and $90 \%$ $\mathrm{VO}_{2 \text { peak }}\left(\mathrm{DP}_{\text {LIN }}\right)$.

\section{Experimental procedures}

The subjects reported to the laboratory two hours before starting the experimental protocol and were fitted with the EMG system and, thereafter, to normalize the amplitudes of the signals obtained, performed maximal voluntary contractions (MVC) with each muscle. Next, they rested in a supine position while the three catheters were inserted under local anesthesia (Carbocain 1\%), utilizing an ultrasound system (Mindray DigiPrince DP-6600, Mindray Bio-Medical Electronics Co., Shenzhen, China) to visualize the vessels, as reported elsewhere (Calbet et al. 2005). For sampling arterial blood, a 16-gauge catheter (Arrow ES-04401, 300 mm, Arrow International Inc., Reading, PA) was inserted percutaneously using the Seldinger technique. Thereafter, a 20-gauge catheter (Arrow ES-04150, $120 \mathrm{~mm}$ ) was inserted into the right femoral vein for collection of venous blood. Finally, another 16-gauge catheter (Arrow ES-04401, $300 \mathrm{~mm}$ ) was inserted into an antecubital vein and then advanced into the subclavian vein for additional sampling of venous blood. (For further details, see Björklund et al. (2010)). All three catheters were sutured to the skin to minimize the risk of movement during exercise and connected to tubing (Alaris G420-B, 2000 mm, Cardinal Health, Switzerland) with a three-way stopcock and filled with isotonic saline containing heparin $\left(100 \mathrm{E} \cdot \mathrm{mL}^{-1}\right)$.

For the actual testing, inclines of $6.5^{\circ}$ and $1^{\circ}$ and velocities calculated to elicit $90 \% \mathrm{VO}_{2 \max }$ and $\mathrm{VO}_{2 \text { peak }}$ were used for DIA $\left(11.2 \pm 0.3 \mathrm{~km} \cdot \mathrm{h}^{-1}\right)$ and DP $(24.3 \pm 1.1$ $\mathrm{km} \cdot \mathrm{h}^{-1}$ ), respectively. The continuous experimental protocol was preceded by a 20 min warm-up with DIA and DP at $60-90 \% \mathrm{VO}_{2 \max } / \mathrm{VO}_{2 \text { peak. }}$ The test itself consisted of 3 min of DIA at $90 \%$ of $\mathrm{VO}_{2 \max }$, followed by 3 min of DP at $90 \% \mathrm{VO}_{2 \text { peak }}$ and, finally, a second 3-min bout of DIA at $90 \% \mathrm{VO}_{2 \max }$.

\section{Physiological measurements}

Respiratory variables were monitored with the mixed expired procedure, employing an ergo-spirometry system (AMIS 2001 model C, Innovision A/S, Odense, Denmark) equipped with pneumotachograph to measure inspiratory flow. This system was calibrated with the standardized procedure described by Björklund et al. $(2007,2010) . \mathrm{VO}_{2}$, $\mathrm{VCO}_{2}$ and the inspired minute ventilation $\left(\mathrm{V}_{\mathrm{E}}\right)$ were monitored continuously and the average $\mathrm{VO}_{2}$ during the final $30 \mathrm{~s}$ at each workload calculated.

Heart rate (HR) was followed continuously using the Polar S610 monitor (Polar Electro Oy, Kempele, Finland) in combination with the metabolic cart. During the final minute of exercise at each bout, arterial and venous blood samples $(1.5 \mathrm{ml})$ were collected anaerobically into syringes (PICO 50, Radiometer, Copenhagen, Denmark) containing heparin and then analyzed immediately for hemoglobin concentration $[\mathrm{Hb}], \mathrm{pH}, \mathrm{PO}_{2}, \mathrm{PCO}_{2}$, oxygen saturation $\left(\mathrm{SO}_{2}\right)$ (ABL 80; Radiometer, Copenhagen, Denmark) and lactate (Biosen 5140, EKF-diagnostic $\mathrm{GmbH}$, Magdeburg, Germany). The $\mathrm{O}_{2}$ content of arterial $\left(\mathrm{CaO}_{2}\right)$ and venous (femoral: $\mathrm{CfvO}_{2}$; subclavian: $\mathrm{CsvO}_{2}$ ) blood was calculated from the $\mathrm{SO}_{2}$ and $[\mathrm{Hb}]$ as follows: $\left(1.34 \times[\mathrm{Hb}] \times \mathrm{SO}_{2}\right)+\left(0.003 \times \mathrm{PO}_{2}\right)($ Calbet et al. 2005) .

\section{Force measurements}

Each participant used custom-made carbon-fiber racing poles adjusted to his preferred length. The ground reaction force was measured by a strain gauge force transducer mounted directly below the grips of these poles (Hottinger-Baldwin Messtechnik GmbH, Darmstadt, Germany). Plantar ski reaction forces were recorded at $100 \mathrm{~Hz}$ by a Pedar mobile system (Novel GmbH, Munich, Germany). These systems for determination of the pole and plantar forces were validated and calibrated utilizing procedures described previously (Holmberg et al. 2005).

\section{EMG measurements}

The EMG activity at the surface of the triceps brachii, latissimus dorsi, rectus abdominis, gluteus maximus, and gastrocnemius (medial head) muscles on the right side of the body were recorded employing pre-gelled bipolar $\mathrm{Ag} / \mathrm{AgCl}$ surface electrodes (Skintact, Leonhard Lang $\mathrm{GmbH}$, Innsbruck, Austria). Prior to fixation of these electrodes, the skin was shaved, abraded lightly, degreased, and disinfected with alcohol. The electrodes were positioned in parallel on the surface of the muscle belly in the direction of the fibers and $30 \mathrm{~mm}$ apart, in accordance with international standards (Hermens et al. 1999). The reference electrode was attached to the tibia. The active and reference electrodes for each muscle were connected to a single differential amplifier (base gain 500; input impedance $>100 \mathrm{M} \Omega$; common mode rejection ratio $>100$ $\mathrm{dB}$; input range $\pm 10 \mathrm{mV}$ ).

\section{Processing the EMG signals}

Prior to the calculation of EMG parameters, the raw signals were band-pass filtered digitally (10-400 Hz; Butterworth $2^{\text {nd }}$ order) to remove both low- and highfrequency noise (Winter 1990). After full wave rectification of the signals the integrated and root mean square electromyography (IEMG and $\mathrm{EMG}_{\mathrm{RMS}}$ respectively) values for defined phases of exercise were calculated for all of these muscles. After training several times, MVC was performed with each muscle (Acierno et al. 1995; 
Holmberg et al. 2005). The recording and processing of MVC data were carried out according to Björklund et al. (2010). Statistical analysis (see further below) for IEMG and $\mathrm{EMG}_{\mathrm{RMS}}$ was performed using the sum, respectively the mean values for the arm (triceps brachii, and latissimus dorsi) and leg muscles (gastrocnemius, gluteus maximus, rectus femoris).

\section{Collection and analysis of biomechanical data}

EMG signals and pole forces were amplified with a telemetric recording system (TeleMyo 2400T G2, Noraxon, Scottsdale, AZ) and simultaneously stored (at a sampling rate of $3000 \mathrm{~Hz}$ ) on a computer via an $\mathrm{A} / \mathrm{D}$ converter card. Synchronization of the measurement of EMG signals and pole and plantar forces was achieved with a signal produced by the Pedar mobile system. The mean values for the ten successive cycles during the final $30 \mathrm{~s}$ of exercise at the three different bouts were subjected to statistical analysis.

Within each cycle of the DIA skiing, the following four phases were analyzed: 1-2) poling and recovery of the arms; 3) leg-gliding (starting from placement of the roller ski and ending when the leg force was minimal immediately prior to the increase in force associated with the push-off); and 4) the push-off and recovery of the legs. One such cycle was defined as extending from a pole plant to the next pole plant on the same side of the body. A cycle of DP skiing was divided into the poling and recovery phases. The average pole and plantar forces were calculated by dividing the integrated signal by the cycle time. All data were processed using the IKE-master software (IKE-Software Solutions, Salzburg, Austria).

\section{Statistical analyses}

All sets of data exhibited a Gaussian distribution, as confirmed by applying the Shapiro-Wilk test. Values are presented as means $\pm \mathrm{SD}$. A two way repeated-measures ANOVA (skiing technique $\times$ sampling site) was employed to test for differences between skiing techniques and sampling sites, as well as between biomechanical parameters associated with the upper- and lower-body. When a significant interaction was observed, paired sampled t-tests or a one-way ANOVA with repeated measures was performed. When only a single measurement at each workload was performed, a one-way ANOVA with repeated measures was used. A Bonferroni Post-Hoc test was performed on global effects identified by ANOVA. Pair-wise relationships between variables were compared using Pearson's product moment correlation coefficient. A level of $\alpha<0.05$ was considered to be statistically significant in all cases. All statistical analyses were performed with the SPSS 18.0 program (IBM Corporation, Somers, NY).

\section{Results}

\section{General observations}

The calculated $\mathrm{VO}_{2}$ for DIA and the averaged measured $\mathrm{VO}_{2}$ during the first and second bout of DIA skiing and were $4.84 \pm 0.95,4.89 \pm 0.13$ and $\mathrm{VO}_{2} 4.99 \pm 0.11$ $1 \cdot \min ^{-1}$, respectively $(P=0.081$ ) (Figure 1$)$. Similarly, the calculated $\mathrm{VO}_{2}$ for DP and the averaged measured $\mathrm{VO}_{2}$ during DP the skiing did not differ $(4.35 \pm 0.36$ and $4.36 \pm 0.49 \mathrm{l} \cdot \mathrm{min}^{-1}$, respectively; $\left.P=0.94\right)$. The cardiorespiratory and biomechanical values are documented in Table 2.

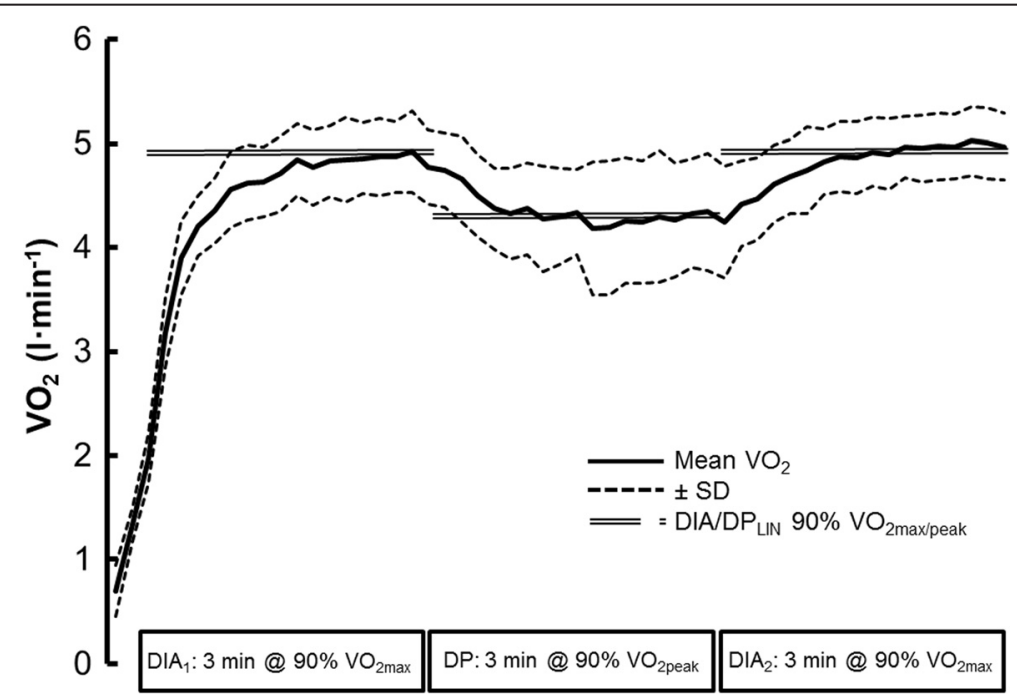

Figure $1 \mathrm{VO}_{2}$ values (means $\pm \mathrm{SD}$ ) during the three sessions of roller skiing on a treadmill. The mean calculated $90 \% \mathrm{VO}_{2 \mathrm{max}}\left(\mathrm{DIA} \mathrm{A}_{\mathrm{LIN}}\right)$ and $90 \% \mathrm{VO}_{2 \text { peak }}\left(\mathrm{DP}_{\mathrm{LIN}}\right)$ are illustrated by the double line. 
Table 2 Cardio-respiratory and biomechanical values for our 8 well-trained male cross-country skiers while roller-skiing with the diagonal stride (DIA) or double poling (DP) technique at $90 \% \mathrm{VO}_{2 \text { max/peak }}$ on a treadmill

\begin{tabular}{|c|c|c|c|c|c|c|}
\hline & First session of DIA & DP & Second session of DIA & $F$-value & $P$-value & Power \\
\hline \multicolumn{7}{|l|}{ Cardio-respiratory parameters } \\
\hline $\mathrm{VO}_{2}\left(1 \cdot \mathrm{min}^{-1}\right)$ & $4.89 \pm 0.36^{\#}$ & $4.36 \pm 0.49^{*}$ & $4.99 \pm 0.11^{\#}$ & $F_{(1.2,8.6)}=35.3$ & $P<0.001$ & $1.000^{\$}$ \\
\hline Heart rate (beats $\cdot \mathrm{min}^{-1}$ ) & $174 \pm 4^{\dagger}$ & $168 \pm 7^{\dagger}$ & $181 \pm 4^{* \#}$ & $F_{(2,14)}=14.7$ & $P<0.001$ & 0.994 \\
\hline$V_{E}\left(\mid \cdot \min ^{-1}\right)$ & $152 \pm 8^{\dagger}$ & $155 \pm 18$ & $172 \pm 14^{*}$ & $F_{(2,14)}=8.52$ & $P=0.004$ & 0.921 \\
\hline $\mathrm{O}_{2}$ pulse (ml per beat) & $28.1 \pm 2.4^{\#}$ & $25.8 \pm 3.1^{*}$ & $27.3 \pm 2.2$ & $F_{(1.1,7.8)}=5.77$ & $P=0.041$ & $0.581^{\$}$ \\
\hline Breaths $\cdot \min ^{-1}$ & $47 \pm 2^{\# \dagger}$ & $52 \pm 3^{*}$ & $53 \pm 7^{*}$ & $F_{(1.2,8.3)}=6.79$ & $P=0.027$ & $0.672^{\$}$ \\
\hline $\mathrm{V}_{T}(\mathrm{ml})$ & $3.24 \pm 0.23^{\#}$ & $2.97 \pm 0.39^{*+}$ & $3.23 \pm 0.27^{\#}$ & $F_{(2,14)}=5.43$ & $P=0.018$ & 0.760 \\
\hline Respiratory exchange ratio & $1.07 \pm 0.06$ & $1.11 \pm 0.06$ & $1.09 \pm 0.04$ & $F_{(2,14)}=1.74$ & n.s. & \\
\hline $\mathrm{V}_{E} \mathrm{NO}_{2}$ & $31.2 \pm 1.9^{\# \dagger}$ & $35.6 \pm 2.1^{*}$ & $34.9 \pm 3.8^{*}$ & $F_{(2,14)}=14.7$ & $P<0.001$ & 0.994 \\
\hline $\mathrm{V}_{E} \mathrm{NCO}_{2}$ & $29.1 \pm 1.1^{\# \dagger}$ & $31.9 \pm 1.4^{*}$ & $32.0 \pm 2.7^{*}$ & $F_{(2,14)}=10.7$ & $P=0.002$ & 0.966 \\
\hline \multicolumn{7}{|l|}{ Biomechanical parameters } \\
\hline Speed $\left(\mathrm{km} \cdot \mathrm{h}^{-1}\right)$ & $11.2 \pm 0.3$ & $24.3 \pm 1.1$ & $11.2 \pm 0.3$ & & & \\
\hline Cycle rate $(\mathrm{Hz})$ & $0.79 \pm 0.02^{\#}$ & $0.87 \pm 0.02^{*+}$ & $0.78 \pm 0.01^{\#}$ & $F_{(2,14)}=13.1$ & $P=0.001$ & 0.985 \\
\hline Cycle length (m) & $3.94 \pm 0.06^{\#}$ & $7.82 \pm 0.23^{*+}$ & $4.00 \pm 0.05^{\#}$ & $F_{(2,14)}=211$ & $P=0.001$ & 1.000 \\
\hline Cycle time (s) & $1.28 \pm 0.07^{\#}$ & $1.16 \pm 0.00^{*+}$ & $1.30 \pm 0.06^{\#}$ & $F_{(2,14)}=12.3$ & $P=0.001$ & 0.979 \\
\hline Recovery time for the arms (s) & $0.75 \pm 0.06^{\#}$ & $0.80 \pm 0.06^{*}$ & $0.75 \pm 0.05^{\#}$ & $F_{(2,14)}=4.91$ & $P=0.028$ & 0.693 \\
\hline Relative arm recovery time (\% cycle time) & $58.7 \pm 2.5^{\#}$ & $68.9 \pm 1.6^{*+}$ & $57.9 \pm 2.7^{\#}$ & $F_{(2,14)}=155$ & $P=0.001$ & 1.000 \\
\hline Peak pole force $(\mathrm{N})$ & $101 \pm 7.4^{\#}$ & $287 \pm 45.2^{* \dagger}$ & $96.0 \pm 5.5^{\#}$ & $F_{(2,14)}=22.3$ & $P=0.001$ & 1.000 \\
\hline Recovery time for the legs (s) & $0.50 \pm 0.04$ & & $0.52 \pm 0.03$ & & n.s. & \\
\hline Peak leg force $(\mathrm{N})$ & $666 \pm 87$ & & $673 \pm 80$ & & n.s. & \\
\hline Impulse of pole force (Ns) & $33.6 \pm 5.9$ & $39.4 \pm 9.6$ & $30.9 \pm 3.7$ & $F_{(2,14)}=4.49$ & $P=0.035$ & 0.651 \\
\hline Impulse of foot force (Ns) & $481 \pm 100$ & $392 \pm 54^{+}$ & $482 \pm 79^{\#}$ & $F_{(2,14)}=9.47$ & $P=0.003$ & 0.937 \\
\hline
\end{tabular}

The values are presented as means $\pm \mathrm{SD}$. $\mathrm{VO}_{2}$, oxygen uptake; $\mathrm{V}_{\mathrm{E}}$, minute ventilation; $\mathrm{V}_{T}$, tidal volume; Bonferroni correction was used to check for differences between time-points. A One-Way repeated ANOVA was used to compare the responses and a paired Student's t-test was when only two time-points were compared. *Statistically different from DIA $_{1}$

"Statistically different from DP.

${ }^{+}$Statistically different from DIA

${ }^{\$}$ Greenhouse-Geisser-adjusted ANOVA, since the sphericity was violated.

n.s. not statistically significant.

\section{Muscle activation}

During the poling phase the $\mathrm{EMG}_{\mathrm{RMS}}$ for the arms was 36.7 and $49.7 \%$ higher with DP than during the first and second sessions of DIA skiing, respectively (both $P<0.05$ ). Especially during DP high $\mathrm{EMG}_{\mathrm{RMS}}$ values of $99.7 \pm 12.9 \% \mathrm{MVC}$ (range: $69-159 \% \mathrm{MVC}$ ) were found. The relative $\mathrm{EMG}_{\mathrm{RMS}}$ for the arms (arms/ whole body) for the entire cycle was $32.7 \%$ lower during DP $(P<0.001)$ than the first session of DIA (Table 3). The sums of the IEMG and average EMG (AEMG) for all of the muscles analyzed during both sessions of DIA were similar and greater than DP (IEMG: DIA 1 : $107 \pm 24$; DP: $86 \pm 26$; $\mathrm{DIA}_{2}$ : $106 \pm 25$ \%MVCs; AEMG: $93 \pm 27$, DP $72 \pm 20$, DIA2 $79 \pm 22 \% \mathrm{MVC} / \mathrm{s} ; P<0.01$ in both cases). The ratio of upper-/whole-body $\mathrm{EMG}_{\mathrm{RMS}}$ was inversely correlated with arm $\mathrm{O}_{2}$ extraction in the arms during both sessions of DIA $(r=-0.918, P=0.004$ and $r=-0.803$, $P=0.03$ respectively) (Figure $2 \mathrm{~A}-\mathrm{B}$ ), whereas there was no such correlation in the case of DP.

\section{Force and kinematics}

Cycle rate and peak pole force were higher during DP than during both sessions of DIA (both $P<0.001$ ), during which these values were similar. The impulse of pole forces was similar in all three sessions $(P>0.05)$. The absolute and relative (\% cycle time) recovery times for the arms were longer with DP, with no difference between the two sessions of DIA (Table 2) $(P>0.05)$. During DIA, $\mathrm{O}_{2}$ extraction in the legs was negatively correlated to minimal leg force during ground contact (the end of the gliding phase) $(r=-0.821, P=0.024)$ and to the IEMG and EMG $\mathrm{RMS}_{\mathrm{RS}}$ for the entire cycle in the lowerbody $(r=-0.782, P=0.038$ and $r=-0.794, P=0.033$, respectively). During DP, $\mathrm{O}_{2}$ extraction in the legs was negatively correlated to minimal leg force $(r=-0.941$, $P=0.002)$ and positively associated with cycle length $(r=0.862, P=0.013) . \mathrm{O}_{2}$ extraction in the arms during DP was correlated to the cycle characteristics length $(r=0.941, P=0.005)$, rate $(r=-0.853, P=0.031)$ and time 
Table 3 The activities of arm and leg muscles during the entire cycle and the poling and recovery phases of the first and second sessions of DIA and the DP skiing at $90 \%$ of $\mathrm{VO}_{2 \text { max/peak }}$

\begin{tabular}{|c|c|c|c|c|c|c|}
\hline & First session of DIA & DP & Second session of DIA & $F$-value & $P$-value & Power \\
\hline IEMG for the entire cycle arms (\%MVCs) & $29.1 \pm 9.2$ & $25.7 \pm 11.1$ & $27.7 \pm 8.0$ & ${ }^{\mathrm{a}} F_{(2,14)}=9.29$ & $P=0.004$ & 0.932 \\
\hline \multirow[t]{2}{*}{ IEMG for the entire cycle legs (\%MVCs) } & $16.1 \pm 3.5^{\#}$ & $11.6 \pm 3.8^{*}$ & $16.0 \pm 3.2^{\#}$ & ${ }^{\mathrm{b}} F_{(1,7)}=15.64$ & $P=0.007$ & 0.906 \\
\hline & & & & ${ }^{c} F_{(2,14)}=0.25$ & n.s & \\
\hline $\mathrm{EMG}_{\mathrm{RMS}}$ poling phase, arms (\%MVC) & $72.9 \pm 8.9^{\#}$ & $99.7 \pm 12.9^{*{ }^{\dagger}}$ & $66.6 \pm 10.7^{\#}$ & ${ }^{\mathrm{a}} F_{(1,7)}=6.66$ & $P=0.042$ & 0.580 \\
\hline \multirow[t]{2}{*}{ EMGRMs push-off phase legs (\%MVC) } & $44.6 \pm 3.5$ & & $43.0 \pm 3.6$ & ${ }^{\mathrm{b}} F_{(1,7)}=7.44$ & $P=0.034$ & 0.626 \\
\hline & & & & ${ }^{c} F_{(1.7)}=1.13$ & n.s. & \\
\hline$E_{\text {MMS }}$ recovery phase, arms (\%MVC) & $14.2 \pm 2.4$ & $14.5 \pm 3.1$ & $15.1 \pm 2.2$ & ${ }^{\mathrm{a}} F_{(2,14)}=39.5$ & $P=0.001$ & 1.000 \\
\hline \multirow[t]{2}{*}{$\mathrm{EMG}_{\mathrm{RMS}}$ recovery phase, legs (\%MVC) } & $31.4 \pm 2.3^{\#}$ & $18.0 \pm 2.8^{* \dagger}$ & $30.5 \pm 2.0^{\#}$ & ${ }^{\mathrm{b}} F_{(2,14)}=51.1$ & $P=0.001$ & 1.000 \\
\hline & & & & ${ }^{c} F_{(4,28)}=5.24$ & $P=0.023$ & 0.723 \\
\hline EMG $_{\text {RMS }}$ ratio, entire cycle, arms/whole body (\%) & $25.1 \pm 1.6^{\#}$ & $16.9 \pm 2.4^{* \dagger}$ & $24.2 \pm 1.5^{\#}$ & $F_{(1,7)}=13.0$ & $P=0.001$ & 0.984 \\
\hline
\end{tabular}

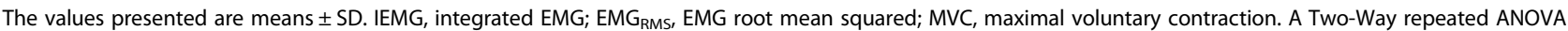
$(3 \times 2)$ was used to compare the responses during the two sessions of DIA and DP. Since there was no push-off phase for the legs during DP, a $2 \times 2$ Two-Way repeated ANOVA was used to compare the EMG RMS for the poling and push-off phases. A One-Way repeated ANOVA was used in the case of the EMG and Bonferroni correction was used to compare different time-points, with paired Student's t-test instead when only two time-points were compared. *Significantly different from $\mathrm{DIA}_{1}$

"Significantly different from DP.

${ }^{\dagger}$ Significantly different from $\mathrm{DIA}_{2}$.

${ }^{a}$ Main effect: exercise intensity.

${ }^{\mathrm{b}}$ Main effect: extremity.

'Interactive effect: exercise intensity $x$ extremity.

n.s. not statistically significant.

$(r=0.865, P=0.026)$, as well as absolute recovery time during the poling phase $(r=0.850, P=0.032)$.

\section{Overall extraction of $\mathrm{O}_{2}$}

$\mathrm{O}_{2}$ extraction was higher in the legs than the arms at all workloads $\left(F_{1,7}=25.9, P=0.002\right)$ and was lower in all limbs during DP than the two session of DIA $\left(F_{2,14}=10.3\right.$, $P=0.002$ and $F_{2,14}=10.4, P=0.002$, respectively), with no difference between the latter (leg $P=1.00$ and arm $P=0.21$ ). There was an interaction between skiing technique and sampling site $\left(F_{2,14}=3.89, P=0.05\right)$, which might reflect the more pronounced reduction in $\mathrm{O}_{2}$ extraction in the arms during DP than both sessions of DIA $\left(F_{2,14}=13.31, P=0.001\right)$ (Figure 3$)$.

\section{Blood levels of lactate and $\mathrm{pH}$}

The arterial-venous difference in blood level of lactate in the legs was lowest (actually negative) during DP and similar during both sessions of DIA $\left(F_{2,14}=5.92\right.$, $P=0.014)$. In contrast, in the case of the arms this difference remained constant $\left(F_{2,14}=2.17, P=0.163\right)$ (Figure 4). The absolute blood level of lactate rose from the first session of DIA to DP and again to the second session of DIA, being significantly higher in the subclavian vein than in either the femoral artery or vein throughout the entire test $(P<0.05)$.

The blood level of bicarbonate fell to an equal extent at all three sampling sites for each successive workload $(P<0.05)$. The $\mathrm{pH}$ was lower in both the subclavian and femoral veins than the femoral artery during all three sessions, with the lowest values for all sampling sites occurring during the second session of DIA $(P<0.01)$. The $\mathrm{PCO}_{2}$ remained constant throughout the test and was higher in both the femoral and subclavian vein than the femoral artery $(P<0.05)$ (Table 4$)$.

\section{The breathing pattern}

During DP the cycle and breathing rates were tightly coupled (i.e. entrainment), with 52 breaths $\cdot \min ^{-1}(0.87 \mathrm{~Hz})$ being similar to the cycle rate (a ratio of 1:1) $(r=0.89$, $P=0.007)$. In the case of DIA, the rate of breathing was similar to the cycle rate during the first session (approximately $0.79 \mathrm{~Hz}$ ), but greater than cycle rate during the second $(0.88 \mathrm{~Hz}$ vs. $0.78 \mathrm{~Hz}, P=0.022)$. Furthermore, there was no correlation between the breathing and cycle rates during either session of DIA $(r=0.23$ and $r=0.18$ both $P>0.05$ ).

\section{Discussion}

The major findings of the study using repeated highintensity DIA bouts interspersed with high-intensity DP were: 1) DP did not alter the redistribution in muscle activation between arms and legs and any further biomechanical variables between $\mathrm{DIA}_{1}$ and $\mathrm{DIA}_{2}$. 2) $\mathrm{O}_{2}$ extraction in both arms and legs was unaffected by DP as $\mathrm{O}_{2}$ extraction was unchanged between DIA $_{1}$ and DIA 2 . However, arm $\mathrm{O}_{2}$ extraction decreased during DP compared to DIA despite higher muscle activation, a 

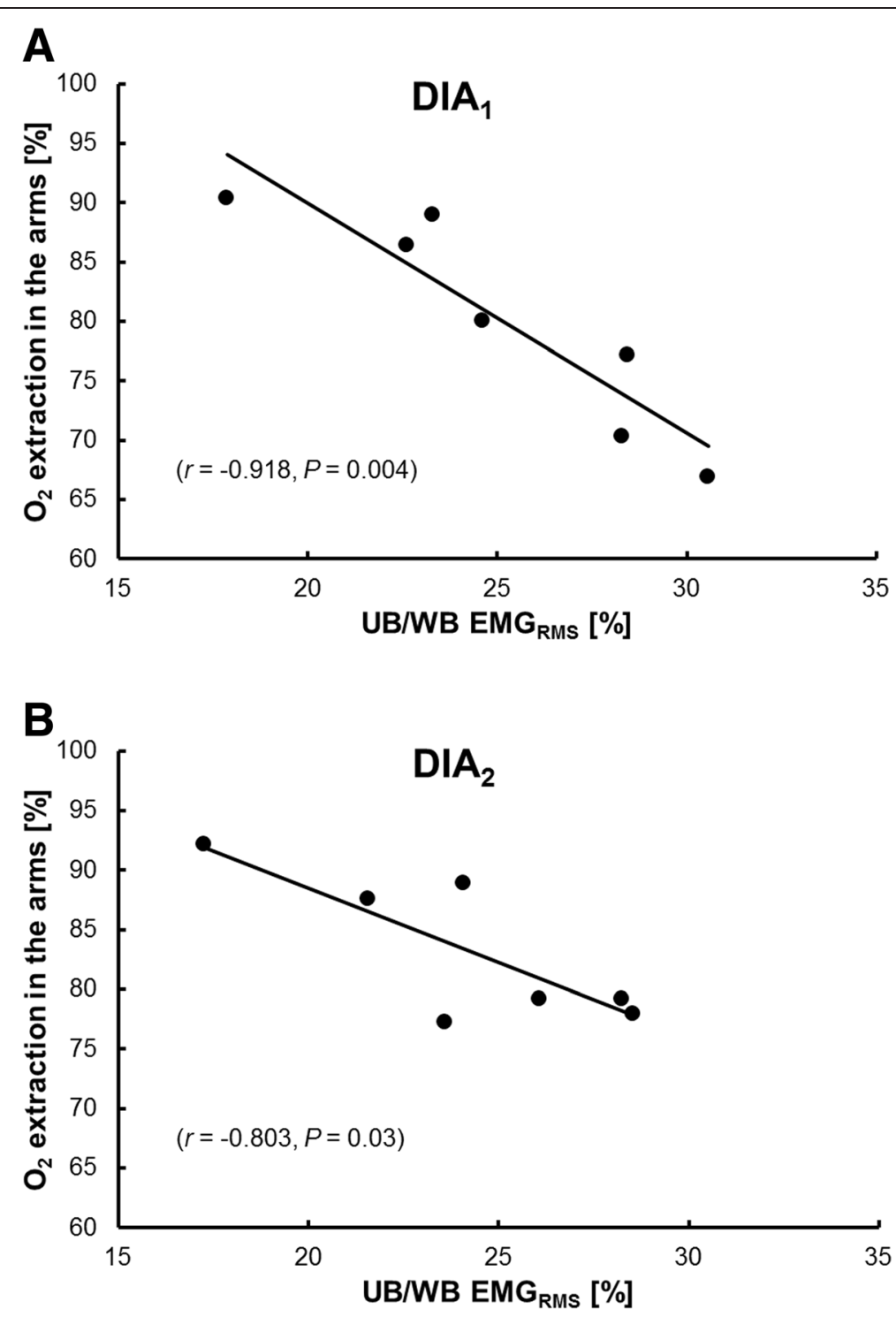

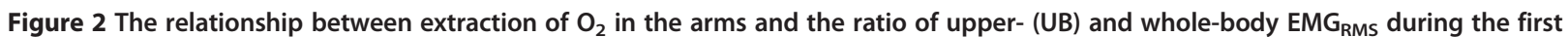
(A) $\left(D_{1} A_{1}\right)$ and second sessions $(B)\left(D I A_{2}\right)$ of diagonal stride skiing.

three-fold increase in pole ground reaction force and an augmented blood lactate concentration in the arms. 3) Leg $\mathrm{O}_{2}$ extraction was related to a greater unloading of the lower body by a more dynamic lower body work during DP and both DIA, while arm $\mathrm{O}_{2}$ extraction was associated with cycle characteristics (e.g. cycle length, cycle rate, swing time) for DP only. Interestingly, a greater ratio of upper- to whole-body muscle activation at both $\mathrm{DIA}_{1}$ and DIA 2 was associated to an attenuated arm $\mathrm{O}_{2}$ extraction. 4) Pulmonary $\mathrm{VO}_{2}$ did not increase over time as $\mathrm{VO}_{2}$ for $\mathrm{DIA}_{1}$ and $\mathrm{DIA}_{2}$ was similar. However, there was an apparent acid base disturbance from $\mathrm{DIA}_{1}$ to $\mathrm{DIA}_{2}$ reflected by a decrease in $\mathrm{pH}$, bicarbonate and an increase in lactate both systemically and for venous arm and leg blood.

\section{Effects of DP on upper to lower body muscle activation} during DIA

Despite increased muscle activation and peak pole forces during DP compared to DIA $(\sim+40 \%,+185 \%)$ no changes in regards of muscle activation distribution between upper- and lower-body and cycle characteristics for $\mathrm{DIA}_{2}$ compared to $\mathrm{DIA}_{1}$ were induced. Previous data from repeated sprint cross-country skiing on snow, using a higher exercise intensity than in the current study, demonstrated a decrease in upper body force and power output during DP (Zory et al. 2009). This decrease was accompanied by a decrease in performance. Our study was carried out on a treadmill using preset velocities where the skier was not able to adjust the velocity. However, this does not explain the absence in redistribution between upper and lower 


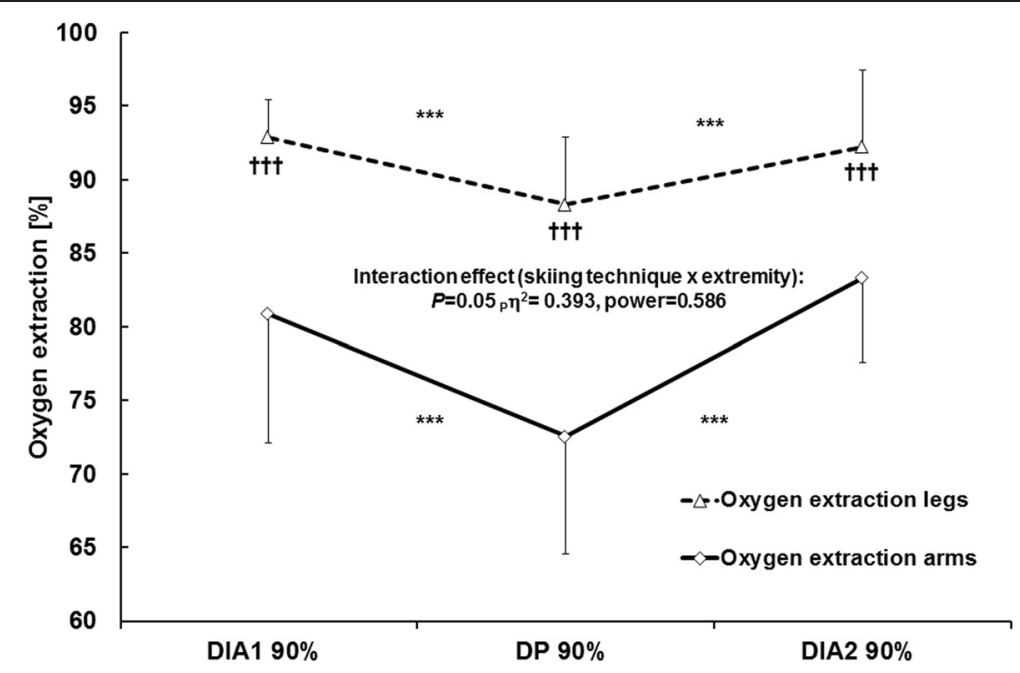

Figure $3 \mathrm{O}_{2}$ extraction in the arms and legs during the first and second sessions of diagonal stride skiing and the intervening double poling at $90 \% \mathrm{VO}_{2 \text { max/peak }}$. The values presented are as means $\pm \mathrm{SD}$. The $P$-values, effect size $\left({ }_{p} \eta^{2}\right)$, and observed power obtained with the two-way ANOVA (skiing technique $\times$ extremity) are presented. ${ }^{* *} P<0.001$ in comparison to DP and $+1+P<0.001$ in comparison to the arms.

body as both arms and legs are used for propulsion during DIA. Also, it could be that the intensity was not strenuous enough for these well-trained skiers in accordance with a previous report that elite athletes withstand fatigue better to maintain neuromuscular control (Bonacci et al. 2011). Furthermore, the increased muscle activation during DP compared to DIA was specifically during the poling phase while for the entire movement cycle as well as the recovery phase no difference was observed. Therefore, even though the high demands on the upper body during highintensity DP the upper-body contribution during DIA seems to be too small to necessitate a redistribution of upper to lower body work.

\section{$\mathrm{O}_{2}$ extraction DP vs. DIA}

One of the novel findings was the decreased arm $\mathrm{O}_{2}$ extraction during DP compared with DIA, although increased upper-body muscle activation (up to $\sim 100 \%$ of MVC in DP vs. $70 \%$ of MVC in DIA). The result of a lower arm $\mathrm{O}_{2}$ extraction during DP than DIA have previously been demonstrated by Calbet et al. (2005) using a lower exercise intensity (76 vs. $90 \%$ of $\mathrm{VO}_{2 \mathrm{max} / \text { peak }}$ ).

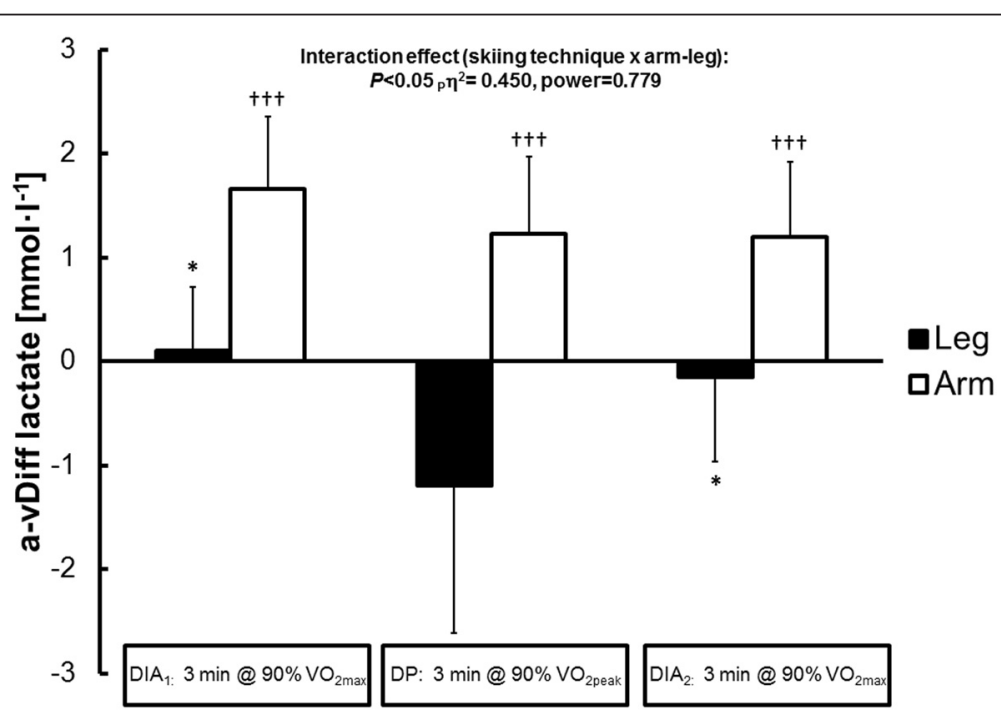

Figure 4 The arterial-venous difference in blood levels of lactate in the legs and arms during the first and second sessions of diagonal stride skiing and the intervening double poling at $\mathbf{9 0} \% \mathbf{V O}_{2 \text { max } / \text { peak }}$. The values presented are means $\pm \mathrm{SD}$. The $P$-values, effect size $\left({ }_{p} \eta^{2}\right)$, and observed power obtained with the two-way ANOVA (skiing technique $x$ extremity) are presented. ${ }^{*} P<0.05$ in comparison to DP and $++\uparrow P<0.001$ in comparison to the legs. 
Table 4 Lactate, pH and gases in the blood during roller-skiing with the diagonal stride (DIA) or double-poling (DP) technique at $90 \% \mathrm{VO}_{2 \text { max/peak }}$

\begin{tabular}{|c|c|c|c|c|c|}
\hline Variable & Resting & First session of DIA & DP & Second session of DIA & $F$-values, $P$-values, observed power \\
\hline \multicolumn{6}{|c|}{ [Lactate], $\mathrm{mmol} \cdot \mathrm{I}^{-1}$} \\
\hline Femoral artery & $0.8( \pm 0.1)$ & $4.6( \pm 0.9)^{\mathrm{a} \dagger}$ & $7.0( \pm 1.6)^{*+}$ & $7.9( \pm 2.0)^{* 0 \dagger}$ & ${ }^{\mathrm{a}} F_{(2,14)}=46.4, P<0.001$, power $=1.0$ \\
\hline Femoral vein & $0.7( \pm 0.2)$ & $4.7( \pm 0.8)^{\mathrm{at}}$ & $5.8( \pm 1.7)^{\dagger}$ & $7.8( \pm 1.8)^{* \mathrm{~g} \dagger}$ & ${ }^{b} F_{(2,14)}=29.4, P<0.001$, power $=1.0$ \\
\hline Subclavian vein & $0.8( \pm 0.3)$ & 6.3. $( \pm 1.4)^{a . j \neq}$ & $8.2( \pm 1.6)^{* \$ \neq}$ & $9.1( \pm 2.0)^{* \xi \neq}$ & ${ }^{c} F_{(4,28)}=5.17, P<0.01$, power $=0.94$ \\
\hline \multicolumn{6}{|c|}{ [Bicarbonate], $\mathrm{mmol} \cdot \mathrm{I}^{-1}$} \\
\hline Femoral artery & $23.6( \pm 1.9)$ & $19.2( \pm 1.0)^{\alpha}$ & $17.8( \pm 1.4)^{*}$ & $16.9( \pm 1.3)^{*}$ & ${ }^{\mathrm{a}} F_{(2,14)}=51.7, P<0.001$, power $=1.0$ \\
\hline Femoral vein & $23.5( \pm 1.6)$ & $19.4( \pm 1.3)^{a}$ & $17.8( \pm 1.6)^{*}$ & $16.6( \pm 1.2)^{* \infty}$ & ${ }^{b} F_{(2,14)}=0.081$, n.s. \\
\hline Subclavian vein & $23.7( \pm 1.3)$ & $19.4( \pm 1.1)^{\alpha}$ & $17.4( \pm 0.8)$ & $16.5( \pm 1.2)^{* \infty}$ & ${ }^{c} F_{(4,28)}=0.212$, n.s. \\
\hline \multicolumn{6}{|l|}{ pH } \\
\hline Femoral artery & $7.42( \pm 0.01)$ & $7.37( \pm 0.02)^{\mathrm{a} \neq \dagger}$ & $7.34( \pm 0.03)^{*+\neq}$ & $7.31( \pm 0.04)^{* \text { of } \neq}$ & ${ }^{\mathrm{a}} F_{(2,14)}=32.97, P<0.001$, power $=1.0$ \\
\hline Femoral vein & $7.38( \pm 0.02)$ & $7.27( \pm 0.02)^{\mathrm{a} \$ \dagger}$ & $7.23( \pm 0.04)^{* \$}$ & $7.19( \pm 0.03)^{* \text { a\$ }}$ & ${ }^{b} F_{(2,14)}=384, P<0.001$, power $=1.0$ \\
\hline Subclavian vein & $7.37( \pm 0.03)$ & $7.24( \pm 0.03)^{a \$ \neq}$ & $7.21( \pm 0.03)^{* \$}$ & $7.19( \pm 0.03)^{* \ldots \$}$ & ${ }^{c} F_{(4,28)}=5.68, P<0.01$, power $=0.95$ \\
\hline \multicolumn{6}{|c|}{$\mathrm{O}_{2}$ content, $\mathrm{ml} \cdot \mathrm{I}^{-1}$} \\
\hline Femoral artery & $190( \pm 15.2)$ & $193( \pm 15.3)^{\dagger \neq}$ & $192( \pm 27.8)^{\dagger \neq}$ & $200( \pm 11.0)^{\dagger \neq}$ & ${ }^{\mathrm{a}} F_{(2,14)}=1.51$, n.s. \\
\hline Femoral vein & $100( \pm 23.9)$ & $13.4( \pm 4.6)^{\text {\$t }}$ & $22.4( \pm 9.9)^{\$+}$ & $15.6( \pm 10.7)^{\text {st }}$ & ${ }^{b} F_{(2,14)}=428, P<0.001$, power $=1.0$ \\
\hline Subclavian vein & $120( \pm 29.8)$ & $36.1( \pm 15.0)^{\text {s }}$ & $51.0( \pm 12.6)^{\$ \neq}$ & $33.2( \pm 11.0)^{\text {\$ }}$ & ${ }^{\mathrm{c}} F_{(4,28)}=2.52$, n.s. \\
\hline \multicolumn{6}{|l|}{$\mathrm{SO}_{2}(\%)$} \\
\hline Femoral artery & $98.3( \pm 0.8)$ & $94.8( \pm 2.0)^{\alpha+\neq}$ & $96.0( \pm 1.7)^{*+\neq}$ & $94.3( \pm 2.0)^{\alpha+\neq}$ & ${ }^{\mathrm{a}} F_{(2,14)}=16.28, P<0.001$, power $=0.99$ \\
\hline Femoral vein & $51.1( \pm 11.1)$ & $6.5( \pm 2.4)^{\mathrm{a} \$ t}$ & $11.2( \pm 5.2)^{* \$+}$ & $7.5( \pm 5.2)^{\mathrm{a} \$ \mathrm{t}}$ & ${ }^{b} F_{(2,14)}=1294, P<0.001$, power $=1.0$ \\
\hline Subclavian vein & $57.0( \pm 17.9)$ & $16.9( \pm 7.5)^{\$ \neq}$ & $22.7( \pm 6.1)^{\$ \neq}$ & $14.7( \pm 5.3)^{a \$ \neq}$ & ${ }^{C} F_{(4,28)}=4.28, P<0.01$, power $=0.87$ \\
\hline \multicolumn{6}{|l|}{$\mathrm{PO}_{2}(\mathrm{~mm} \mathrm{Hg})$} \\
\hline Femoral artery & $111( \pm 15.2)$ & $77.3( \pm 10.8)^{a+\neq}$ & $88.1( \pm 11.4)^{*+\neq}$ & $78.9( \pm 9.8)^{\mathrm{a} \dagger \neq}$ & ${ }^{\mathrm{a}} F_{(2,14)}=15.47, P<0.001$, power $=0.99$ \\
\hline Femoral vein & $27.5( \pm 4.8)$ & $8.8( \pm 2.3)^{\mathrm{a} \$+}$ & $12.6( \pm 3.6)^{* \$ \dagger}$ & $9.8( \pm 4.5)^{\mathrm{a} \$}$ & ${ }^{b} F_{(2,14)}=471, P<0.001$, power $=1.0$ \\
\hline Subclavian vein & $32.1( \pm 8.7)$ & $16.3( \pm 4.2)^{\$ \neq}$ & $20.0( \pm 3.2)^{\$ \neq}$ & $15.8( \pm 3.7)^{\$ \neq}$ & ${ }^{C} F_{(4,28)}=4.21, P<0.05$, power $=0.86$ \\
\hline \multicolumn{6}{|l|}{$\mathrm{PCO}_{2}(\mathrm{~mm} \mathrm{Hg})$} \\
\hline Femoral artery & $34.9( \pm 4.0)$ & $30.5( \pm 2.6)^{\dagger \neq}$ & $29.8( \pm 2.3)^{\dagger \neq}$ & $29.3( \pm 2.3)^{\dagger \neq}$ & ${ }^{\mathrm{a}} F_{(2,14)}=2.04$, n.s. \\
\hline Femoral vein & $43.5( \pm 4.2)$ & $55.1( \pm 4.1)^{5}$ & $54.8( \pm 3.2)^{\$}$ & $59.0( \pm 4.9)^{5}$ & ${ }^{b} F_{(2,14)}=179, P<0.001$, power $=1.0$ \\
\hline Subclavian vein & $45.5( \pm 5.3)$ & $60.3( \pm 7.0)^{\$}$ & $57.9( \pm 5.1)^{\$}$ & $59.8( \pm 2.9)^{\$}$ & ${ }^{c} F_{(4,28)}=2.26$, n.s. \\
\hline
\end{tabular}

The values shown are means $( \pm \mathrm{SD}) . \mathrm{PO}_{2}$, partial pressure of oxygen; $\mathrm{PCO}_{2}$, partial pressure of carbon dioxide; $\mathrm{SO}_{2}$, oxygen saturation.

The $F$ - and $P$-values are for two-way ANOVA $(3 \times 3)$ analysis of exercise intensity $\left(90 \% \mathrm{VO}_{2 \text { max/peak }}\right) \times$ sampling site.

a Main effect between intensities. ${ }^{\$}$ Significantly different to femoral artery. ${ }^{*}$ Significantly different to $\mathrm{DIA}_{1} 90 \%$.

${ }^{b}$ Main effect between sampling sites. ${ }^{\ddagger}$ Significantly different to femoral vein. ${ }^{\text {a }}$ Significantly different to DP90\%.

Intensity by sampling site interactive effect. ${ }^{\dagger}$ Significantly different to subclavian vein.

n.s. not significant.

Furthermore, there was an interaction effect caused by a more pronounced decrease in $\mathrm{O}_{2}$ extraction in arms than legs when switching from DIA ${ }_{1}$ to DP and back to DIA $_{2}$. The reason for a lower arm $\mathrm{O}_{2}$ extraction during DP has been shown, at least in part, to be due to a possible mechanical hindrance caused by high pole force generation and high arm muscle activity ( 96\% MVC) that hinders further elevation of $\mathrm{O}_{2}$ extraction (Stöggl et al. 2013). The current study supports this finding, based on the reduction in arm $\mathrm{O}_{2}$ extraction despite increased pole forces and upper body muscle activation when comparing DP with DIA.
Another explanation for the attenuated arm $\mathrm{O}_{2}$ extraction during DP could be the decrease in total activated muscle mass, as reflected by the increased sum IEMG and AEMG values during DIA compared to DP. Mortensen et al. (2008) demonstrated that when activating a larger muscle mass (two-legged supra maximal cycling compared with one-legged exercise), locomotor skeletal muscle perfusion leveled off with a concomitant larger increase in $\mathrm{O}_{2}$ extraction. Furthermore, comparisons between arm cycling vs. combined arm and leg cycling shows a decrease in arm blood flow when adding leg to arm exercise but at the same time increased $\mathrm{O}_{2}$ extraction 
in the arms (Volianitis and Secher 2002). More specifically, in a study by Calbet et al. (2004) arm blood flow was substantially higher using DP than DIA although at a similar cardiac output. Therefore, in the current study high-intensity DIA likely induce additional central cardiovascular stress due to an increased activated total muscle mass than DP - as demonstrated by the augmented sum IEMG and AMEG values - and thus induces a higher $\mathrm{O}_{2}$ extraction in both arms and legs to match the increased $\mathrm{O}_{2}$ demand.

\section{Relationships between biomechanical variables and $\mathrm{O}_{2}$ extraction}

Skiers who unloaded their legs to a greater extent (leg force minima) at the end of the gliding phase prior to leg push-off during DIA and prior to pole plant during DP extracted more $\mathrm{O}_{2}$ in the legs. The last finding is in accordance with a previous study from our group were leg force minima was related to both $\mathrm{O}_{2}$ extraction in the legs and arms at $90 \%$ and $70 \%$ of $\mathrm{VO}_{2 \text { peak }}$ during DP (Stöggl et al. 2013). Furthermore, this result fits nicely with the previous demonstration that modern DP involves more dynamic use of the lower body: a higher body position prior to pole plant; greater extension of the knee, hip and ankle joints; and distinct knee and hip flexion during the poling phase (Holmberg et al. 2005). In addition Stöggl et al. (2010) demonstrated that faster skiers had greater center of mass oscillation in vertical direction during DP and DIA being coupled with a more active and dynamic skiing technique. The aspect about coupling between greater unloading of the legs prior to the push-off phase during DIA and leg $\mathrm{O}_{2}$ extraction is novel. Therefore, more technically skilled skiers with more active and dynamic technique achieve a better $\mathrm{O}_{2}$ extraction.

Arm $\mathrm{O}_{2}$ extraction was negatively associated to cycle rate and positively related to cycle length and arm recovery time during DP, being in line with our previous findings during DP (Stöggl et al. 2013). This association between the decreased arm $\mathrm{O}_{2}$ extraction and cycle rate during DP might be influenced by coupling to limb blood flow. Accordingly, an increased cycle rate might increase the centrifugal force that amplifies the arm blood flow as shown in another study using arm exercise at a similar cycle rate as in the current study $(0.75 \mathrm{~Hz})$ (Sheriff et al. 2009). There is further support that an increased contraction frequency (in our case cycle rate) induces a greater muscle blood flow (Ferguson et al. 2001; Sheriff 2003). Possibly the increased cycle rate could induce a higher arterial pressure that increase or maintains a high blood flow although usually such a high muscle activity shown in the current study restricts blood flow (70-100\% of MVC). For the legs on the other hand no relationship was noticed between cycle rate and leg $\mathrm{O}_{2}$ extraction which is in accordance with previous findings using isolated leg exercise (Ferguson et al. 2001).

\section{Excess $\mathrm{VO}_{2}$, muscle activation and lactate}

It is suggested that it is not possible to perform a constant work rate at higher intensities that refers to a specific percent of $\mathrm{VO}_{2 \max }$ (Whipp 1994). One of the mechanisms proposed for this $\mathrm{VO}_{2}$ slow component is the magnitude and the time course of the increase in blood lactate, i.e. above the lactate threshold (Burnley and Jones 2007). In contrast to that, in the current study no changes in $\mathrm{VO}_{2}$ were found between $\mathrm{DIA}_{1}$ and $\mathrm{DIA}_{2}$ even though blood lactate steadily increased throughout the protocol (arterial: 4.6 to $7.9 \mathrm{mmol} \cdot \mathrm{l}^{-1}$ ). Comparable results were found in the study of Björklund et al. (2011) also demonstrating no increase in $\mathrm{VO}_{2}$ in world class cross-country skiers, when utilizing a variable intensity protocol containing 3 minutes exercise intensity of $90 \%$ of $\mathrm{VO}_{2 \max }$ interspersed with 6 minutes of $70 \%$ of $\mathrm{VO}_{2 \max }$ with a total duration of $24 \mathrm{~min}$. One reason for the absence of a raise in $\mathrm{VO}_{2}$ from $\mathrm{DIA}_{1}$ to $\mathrm{DIA}_{2}$ in the current study might be due to the large amount of activated muscle mass and also already high $\mathrm{O}_{2}$ extraction ( $90 \%)$ with DIA and DP making the potential for a further increase in $\mathrm{VO}_{2}$ diminutive.

\section{Respiration locomotion coordination}

While the exercise intensity was similar between the two DIA bouts there was a shift in breathing pattern. First, $\mathrm{V}_{E}$ increased for the second DIA due to an increase in $\mathrm{B}_{f}$ ( 47 vs. 53 breaths $\cdot \min ^{-1}$ ) while no change in tidal volume. This is in accordance with previously shown data using DIA although at a lower exercise intensity $\left(\sim 76 \% \mathrm{VO}_{2 \max }\right)$ (Holmberg and Calbet 2007). Second, during the first DIA the $\mathrm{B}_{f}$ was similar as cycle rate (approximately $0.79 \mathrm{~Hz}$ ), but at the second DIA workload there was a tachypneic shift, due to the elevated $\mathrm{V}_{E}$ caused by an increased $\mathrm{B}_{f}$ with a mean ratio of $1.13: 1$, (range $1 .: 1-1.4: 1)\left(\mathrm{B}_{f} 0.88 \mathrm{~Hz}\right.$ vs. cycle rate $0.78 \mathrm{~Hz}$ ). The cause for this asynchronic $\mathrm{B}_{f}$ to cycle rate ratio during $\mathrm{DIA}_{2}$ could be explained by the increased metabolic acidosis as the arterial blood lactate concentration went from 4.6 to $7.9 \mathrm{mmol} \cdot \mathrm{l}^{-1}$ and the $\mathrm{pH}$ dropped from 7.37 to 7.31 between $\mathrm{DIA}_{1}$ to $\mathrm{DIA}_{2}$. However the skiers $\mathrm{P}_{\mathrm{a}} \mathrm{CO}_{2}$ remained fairly stable and did not show signs of hypercapnia, nor was there a relation between the magnitude of asynchronic breathing and $\mathrm{P}_{\mathrm{a}} \mathrm{CO}_{2}$. Further, even though $\mathrm{B} f$ increased between $\mathrm{DIA}_{1}$ and $\mathrm{DIA}_{2}$ the $\mathrm{B} f$ remained unchanged between DP and DIA although the latter of the two was performed with a lower cycle rate. The coordination between respiration - locomotion was therefore not fixed for DIA. However the breathing pattern in the current study confirms that during the single bout of DP there was a tight coupling between cycle rate and $\mathrm{B}_{f}$, i.e. entrainment, with a $\mathrm{B}_{f}$ of 52 breaths $\cdot \min ^{-1}$ equaling $0.87 \mathrm{~Hz}$ being similar to the cycle 
rate in DP (1:1). This 1:1 coupling could be exercise intensity dependent as shown previously during DP using a fixed cycle rate of $60 \mathrm{~Hz}$ were $\mathrm{B}_{f}$ and cycle rate was uncoordinated for lower speeds $\left(12-18 \mathrm{~km} \cdot \mathrm{h}^{-1}\right)$ while attaining a $1: 1$ ratio during $24 \mathrm{~km} \cdot \mathrm{h}^{-1}$ (Lindinger and Holmberg 2011).

\section{Limitations}

Due to the lack of blood flow measurements it cannot be definitively concluded whether the differences in $\mathrm{O}_{2}$ extraction between the arms and legs and between DP and DIA are due to differences in blood flow and/or metabolic demands. Further arterial blood pressure measurements could add information regarding the effect of arm swing and cycle rate influence of both $\mathrm{O}_{2}$ extraction and blood flow. This link was especially pronounced during mainly upper-body work, i.e. DP in the current study.

\section{Conclusions}

In well trained skiers, DP does not influence DIA $\mathrm{VO}_{2}$, at a simulated race intensity of $\sim 90 \%$ of $\mathrm{VO}_{2 \max / \text { peak }}$. Furthermore, although high-intensity DP induce substantially higher arm muscle activation concurrent with a three-fold increase in pole force compared to DIA, it does not affect the redistribution of pole and foot forces, cycle characteristics and cardiorespiratory characteristics during the following DIA bout. This suggests that well trained skiers withstand the upper-body strain that highintensity DP implies. The absence of an increase in $\mathrm{VO}_{2}$ from $\mathrm{DIA}_{1}$ to $\mathrm{DIA}_{2}$ despite a distinct increase of blood lactate questions the relation between blood lactate and excess $\mathrm{VO}_{2}$, and also strengthens the idea that $\mathrm{VO}_{2}$ slow component might not occur in well-trained athletes when using exercise modes where both legs and arms are at their upper limit of $\mathrm{O}_{2}$ extraction. Furthermore, the increase in blood lactate with no change in $\mathrm{VO}_{2}$ and measured biomechanical variables questions that lactate sampling from either leg or arm is an adequate method to establish arm or leg muscle activation during crosscountry skiing. Furthermore, arm $\mathrm{O}_{2}$ extraction decreased during DP compared to DIA although both an increased pole force and muscle activation, pointing towards mechanical hindrance of blood flow during DP. Finally a greater ratio of upper- to lower-body muscle activation was associated with an attenuated arm $\mathrm{O}_{2}$ extraction in DIA, while increased cycle length, paralleled with increased recovery time and more active and dynamic lower body use increases $\mathrm{O}_{2}$ extraction especially in DP.

\section{Abbreviations}

AEMG: Average electromyography; $\mathrm{CaO}_{2}: \mathrm{O}_{2}$ content of arterial blood; $\mathrm{CfvO}_{2}$ : Oxygen content of venous femoral blood; $\mathrm{CsvO}_{2}$ : Oxygen content of subclavian femoral blood; DIA: Diagonal stride; DIA achieving $90 \%$ of $\mathrm{VO}_{2 \max }$ during DIA; DP: Double poling; DP LIN: Workload required achieving $90 \%$ of $\mathrm{VO}_{\text {2peak }}$ during DP; $\mathrm{EMG}_{\mathrm{RMs}}$ : Root mean square electromyography; HR: Heart rate; $\mathrm{HR}_{\text {max: }}$ : Maximal heart rate; IEMG: Integrated electromyography; MVC: Maximal voluntary contraction; OBLA: Onset of blood lactate accumulation; $\mathrm{SO}_{2}$ : Oxygen saturation; $\mathrm{VCO}_{2}$ : Carbone dioxide production; $\mathrm{V}_{\mathrm{E}}$ : Minute ventilation; $\mathrm{VO}_{2}$ : Oxygen uptake; $\mathrm{VO}_{2 \text { max }}$ : Maximal oxygen uptake; $\mathrm{VO}_{\text {2peak: }}$ Peak oxygen uptake.

\section{Competing interests}

The authors declare that they have no competing interests.

\section{Authors' contributions}

$\mathrm{GB}$ and $\mathrm{HH}$ participated in the critical conception and design of the work; GB and TS performed the acquisition of data and analysis (physiological and biomechanical data respectively); GB, HH and TS performed the interpretation of data, drafting the work and revising it critically for important intellectual content and final approval of the version to be published and agreement to be accountable for all aspects of the work.

\section{Acknowledgements}

The authors thank the athletes and the trainers involved in this study for their participation, enthusiasm, and cooperation. This investigation was financially supported by the Swedish National Centre of Research in Sports (CIF).

\section{Author details}

'Department of Health Sciences, Swedish Winter Sports Research Centre, Mid-Sweden University, 83125 Östersund, Sweden. ${ }^{2}$ Swedish Olympic Committee, Stockholm, Sweden. ${ }^{3}$ Department of Sport Science and Kinesiology, University of Salzburg, Salzburg, Austria.

Received: 3 December 2014 Accepted: 5 January 2015

Published online: 01 February 2015

\section{References}

Acierno SP, Baratta RV, Solomonow M (1995) A practical guide to electromyography for biomechansists. Louisiana State University, New Orleans

Ahlborg G, Jensen-Urstad M (1991) Metabolism in exercising arm vs. leg muscle. Clin Physiol 11(5):459-468

Björklund G, Pettersson S, Schagatay E (2007) Performance predicting factors in prolonged exhausting exercise of varying intensity. Eur J Appl Physiol 99(4):423-429

Björklund G, Stöggl T, Holmberg HC (2010) Biomechanically influenced differences in $\mathrm{O} 2$ extraction in diagonal skiing: arm versus leg. Med Sci Sports Exerc 42(10):1899-1908, doi:10.1249/MSS.0b013e3181da4339

Björklund G, Laaksonen MS, Holmberg HC (2011) Blood lactate recovery and respiratory responses during diagonal skiing of variable intensity. Eur J Sport Sci 11(5):317-326

Bonacci J, Green D, Saunders PU, Blanch P, Franettovich M, Chapman AR, Vicenzino $B$ (2010) Change in running kinematics after cycling are related to alterations in running economy in triathletes. J Sci Med Sport 13(4):460-464

Bonacci J, Saunders PU, Alexander M, Blanch P, Vicenzino B (2011) Neuromuscular control and running economy is preserved in elite international triathletes after cycling. Sports Biomech 10(1):59-71

Burnley M, Jones AM (2007) Oxygen uptake kinetics as a determinant of sports performance. Eur J Sport Sci 7(2):63-79, doi:10.1080/17461390701456148

Calbet JA, Jensen-Urstad M, van Hall G, Holmberg HC, Rosdahl H, Saltin B (2004) Maximal muscular vascular conductances during whole body upright exercise in humans. J Physiol 558(Pt 1):319-331

Calbet JA, Holmberg HC, Rosdahl H, van Hall G, Jensen-Urstad M, Saltin B (2005) Why do arms extract less oxygen than legs during exercise? Am J Physiol Regul Integr Comp Physiol 289(5):R1448-R1458

Ferguson RA, Ball D, Krustrup P, Aagaard P, Kjaer M, Sargeant AJ, Hellsten Y, Bangsbo I (2001) Muscle oxygen uptake and energy turnover during dynamic exercise at different contraction frequencies in humans. J Physio 536(Pt 1):261-271, doi:PHY_12634

Hermens HJ, Freriks B, Merletti R, Stegeman DF, Blok JH, Rau G, Disselhorst-Klug C, Hägg G (1999) European recommendations for surface electromyography results of the SENIAM project. Roessingh research and development, Enschede 
Holmberg HC, Calbet JA (2007) Insufficient ventilation as a cause of impaired pulmonary gas exchange during submaximal exercise. Respir Physiol Neurobiol 157(2-3):348-359

Holmberg HC, Lindinger S, Stöggl T, Eitzlmair E, Müller E (2005) Biomechanical analysis of double poling in elite cross-country skiers. Med Sci Sports Exerc 37(5):807-818, doi:00005768-200505000-00015

Holmberg HC, Lindinger S, Stöggl T, Björklund G, Müller E (2006) Contribution of the legs to double-poling performance in elite cross-country skiers. Med Sci Sports Exerc 38(10):1853-1860, doi:00005768-200610000-00022

Ivy JL, Withers RT, Van Handel PJ, Elger DH, Costill DL (1980) Muscle respiratory capacity and fiber type as determinants of the lactate threshold. J Appl Physiol 48(3):523-527

Larsson P, Henriksson-Larsen K (2005) Combined metabolic gas analyser and dGPS analysis of performance in cross-country skiing. J Sports Sci 23(8):861-870

Lindinger SJ, Holmberg HC (2011) How do elite cross-country skiers adapt to different double poling frequencies at low to high speeds? Eur J Appl Physio 111(6):1103-1119, doi:10.1007/s00421-010-1736-8

Lindinger SJ, Göpfert C, Stöggl T, Müller E, Holmberg HC (2009) Biomechanical pole and leg characteristics during uphill diagonal roller skiing. Sports Biomech 8(4):318-333, doi:10.1080/14763140903414417

Mognoni P, Rossi G, Gastaldelli F, Canclini A, Cotelli F (2001) Heart rate profiles and energy cost of locomotion during cross-country skiing races. Eur J Appl Physiol 85(1-2):62-67

Mortensen SP, Damsgaard R, Dawson EA, Secher NH, Gonzalez-Alonso J (2008) Restrictions in systemic and locomotor skeletal muscle perfusion, oxygen supply and VO2 during high-intensity whole-body exercise in humans. J Physiol 586(10):2621-2635, doi:jphysiol.2007.149401

Mygind E, Andersen LB, Rasmussen B (1994) Blood lactate and respiratory variables in elite cross-country skiing at racing speeds. Scand J Med Sc Sports 4(4):243-251, doi:10.1111/j.1600-0838.1994.tb00435.x

Ortenblad N, Nielsen J, Saltin B, Holmberg HC (2011) Role of glycogen availability in sarcoplasmic reticulum $\mathrm{Ca} 2+$ kinetics in human skeletal muscle. J Physiol 589(Pt 3):711-725, doi:jphysiol.2010.195982

Rud B, Secher NH, Nilsson J, Smith G, Hallen J (2014) Metabolic and mechanical involvement of arms and legs in simulated double pole skiing. Scand J Med Sci Sports 24(6):913-919, doi:10.1111/sms.12133

Sheriff DD (2003) Muscle pump function during locomotion: mechanical coupling of stride frequency and muscle blood flow. Am J Physiol Heart Circ Physiol 284(6 53-6):H2185-H2191

Sheriff DD, Mullin TM, Wong BJ, Ladouceur M (2009) Does limb angular motion raise limb arterial pressure? Acta Physiol 195(3):367-374

Sjödin B, Jacobs I (1981) Onset of blood lactate accumulation and marathon running performance. Int J Sports Med 2(1):23-26

Stöggl T, Enqvist J, Müller E, Holmberg HC (2010) Relationships between body composition, body dimensions, and peak speed in cross-country sprint skiing J Sports Sci 28(2):161-169, doi:10.1080/02640410903414160

Stöggl T, Björklund G, Holmberg HC (2013) Biomechanical determinants of oxygen extraction during cross-country skiing. Scand J Med Sci Sports 23(1):e9-e20, doi:10.1111/sms.12004

Van Hall G, Jensen-Urstad M, Rosdahl H, Holmberg HC, Saltin B, Calbet JA (2003) Leg and arm lactate and substrate kinetics during exercise. Am J Physiol Endocrinol Metab 284(1):E193-E205

Volianitis S, Secher NH (2002) Arm blood flow and metabolism during arm and combined arm and leg exercise in humans. J Physiol 544(Pt 3):977-984, doi:PHY_023556

Welde B, Evertsen F, Von Heimburg E, Ingulf Medbo J (2003) Energy cost of free technique and classical cross-country skiing at racing speeds. Med Sci Sports Exerc 35(5):818-825

Whipp BJ (1994) The slow component of $\mathrm{O}_{2}$ uptake kinetics during heavy exercise. Med Sci Sports Exerc 26(11):1319-1326

Winter DA (1990) Biomechanics and motor control of human movement, 2nd edn. John Wiley \& Sons, New York

Zory R, Vuillerme N, Pellegrini B, Schena F, Rouard A (2009) Effect of fatigue on double pole kinematics in sprint cross-country skiing. Hum Mov Sci 28(1):85-98

Zory R, Molinari F, Knaflitz M, Schena F, Rouard A (2011) Muscle fatigue during cross country sprint assessed by activation patterns and electromyographic signals time-frequency analysis. Scand J Med Sci Sports 21(6):783-790

\section{Submit your manuscript to a SpringerOpen ${ }^{\odot}$ journal and benefit from:}

- Convenient online submission

Rigorous peer review

- Immediate publication on acceptance

- Open access: articles freely available online

- High visibility within the field

- Retaining the copyright to your article

Submit your next manuscript at $>$ springeropen.com 